\title{
Perioperative management of malignant pheochromocytoma with coronary artery disease- A case report
}

\author{
Sambhunath Das ${ }^{1}$ \\ ${ }^{1}$ Professor, Dept. of Cardiothoracic Anaesthesia, All India Institute of Medical Sciences, New Delhi, India
}

*Corresponding Author: Sambhunath Das

Email: sambhunathds833@gmail.com

\begin{abstract}
Presence of malignant pheochromocytoma tumor and coronary artery disease is extremely rare and carry high perioperative risk. The postoperative morbidity and mortality are increased by many folds. In the reference case, adrenal tumor had infiltrated to inferior vena cava (IVC), liver and right kidney. He had associated coronary artery disease. We describe the management of a patient with malignant tumor of adrenal gland undergoing surgical removal with all arrangements for coronary artery bypass grafting and intrathoracic extraction of tumor from IVC. It is a rare example of cardiac patient for non-cardiac surgery.
\end{abstract}

Keywords: Cardiac patient for non-cardiac surgery, Coronary artery disease, Malignant pheochromocytoma, Pheochromocytoma.

\section{Introduction}

Combination of malignant pheochromocytoma (PCC) tumor and coronary artery disease (CAD) is extremely rare and carry high perioperative risk. ${ }^{1}$ The postoperative morbidity and mortality are increased by many folds. In the reference case, adrenal tumor had infiltrated to inferior vena cava (IVC), liver and right kidney. He had associated CAD. The development of perioperative myocardial ischemia (MI) is a common incidence with CAD during PCC surgery. We describe the management of a patient with malignant tumor of adrenal gland undergoing surgical removal with all arrangements for coronary artery bypass grafting (CABG) and intrathoracic extraction of tumor from IVC by cardiopulmonary bypass (CPB) support. It is an example of cardiac patient for non-cardiac surgery. ${ }^{2}$

\section{Case Description}

A 57 year male $53 \mathrm{Kg}$ weight with symptoms of head ache, palpitation and episodes of sweating was diagnosed of malignant adrenal tumor or otherwise called pheochromocytoma and CAD. The malignant tumor was abutting into inferior vena cava (IVC). The left anterior descending (LAD) coronary artery lumen was 60\% occluded by atherosclerosis.

The laboratory investigations revealed hemoglobin of 9gm\%; normal platelet, leucocyte and RBC counts. The creatinine was $1.8 \mathrm{mg} / \mathrm{dL}$, urea $44 \mathrm{mg} / \mathrm{dL}$. Liver enzymes were within normal range. The recent reports of PCC like plasma and urinary catecholamine parameters were normal and histology of metastatic lymph nodes revealed nonsecreting tumor with malignant cells. Blood pressure was controlled with alpha and beta-blocker. The CT scan abdomen of patient detected tumor of $10 \times 10 \mathrm{~cm}$ in the adrenal area with extension into IVC, portal vein and inferior surface of liver. Tumor had displaced the right kidney downwards and laterally. Coronary angiography revealed significant disease of LAD.

Surgery was planned as semi-urgent in the cardiothoracic vascular surgery operation room due to the anticipation of establishing CPB and coronary artery bypass grafting $(\mathrm{CABG})$ in case of emergency hemodynamic instability, intraoperative $\mathrm{MI}$ and invasion of tumor into IVC. Premedication of morphine, promethergine, medications related to PCC and CAD; and overnight fasting were advised to the patient. General anaesthesia was induced with trachea-bronchial intubation. Fentanyl, midazolam, dexmedetomidine, propofol and rocuronium were used to induce anesthesia. Esmolol and lignocaine were used to attenuate the sympathetic response during laryngoscopy and tracheal intubation. Double lumen tube was inserted to facilitate single lung ventilation in the need of thoracotomy to surgically approach intra-thoracic part of IVC. Monitoring in the form of ECG, SPO2, ETCO2, invasive blood pressure (IBP), central venous pressure (CVP), bispectral index (BIS), transesophageal echocardiography (TEE), temperature, arterial blood gas, blood sugar and urine output was used during surgery. Transesophageal echocardiography examination revealed normal ventricular function normal valves and invasion of tumor into IVC (Fig. 1). The patient was mechanically ventilated with O2-air-sevoflurane-fentanyl-midalazolam and cisatracurium.

Midline laparotomy approach was used to reach the tumor, while $\mathrm{CPB}$ and CABG were kept on standby. Extensive surgical dissection was performed to expose the malignant adrenal tumor (Fig. 2). The tumor was encroaching walls of IVC but not invaded into the lumen. The tumor had adhered to portal vein, liver and right kidney. Still with patience resection the whole tumor of $10 \times 10 \mathrm{~cm}$ size excised out surgically. The blood loss was replaced with fluid and blood. Epsilon aminocaproic acid was used to restrict bleeding from fibrinolysis. The temperature of the patient was maintained with regulation of ambient temperature, warming blanket and fluid warmer. Surgery continued for 4.5 hour. The hemodynamic parameters were stabilized with dexmedetomidine, nitroglycerine and noradrenaline infusion as per necessary and no episodes of ECG changes and regional wall motion abnormality (RWMA) was detected in TEE. Reassessment of TEE 
revealed no tumor mass inside IVC. Hence CPB and CABG were not needed at this stage.

The patient was shifted to ICU for stabilization of vitals and observation. No major fluctuation of blood pressure and ECG changes were observed for 6hrs. The patient was weaned and extubated after 8hrs. Post-extubation period was uneventful. Patient was discharged from ward after 8 days for chemotherapy.

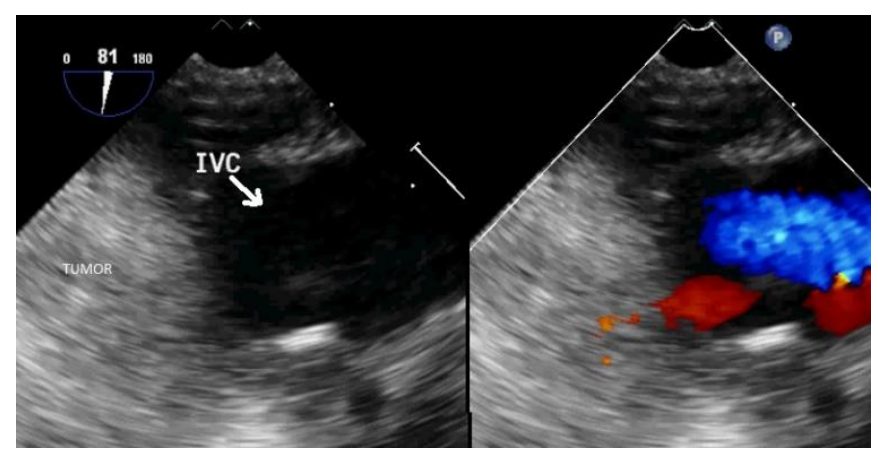

Fig. 1: Transesophageal echocardiography midesophageal at $80^{\circ}$ angle curser inferior vena cava- hepatic vein color compare view showing the tumor abutting to IVC. IVC= inferior vena cava.

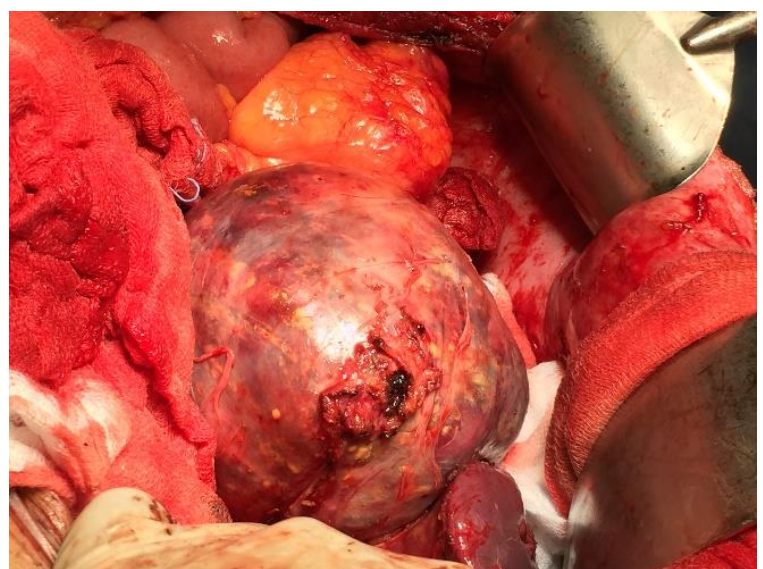

Fig. 2: Intraoperative picture of the huge malignant pheochromocytoma tumor

\section{Discussion}

The patient had malignant PCC with prior diagnosis of vascular invasion and CAD. Despite high surgical risk the case was successfully managed with extensive planning and team work. There was no perioperative MI and major hemodynamic alteration.

Pheochromocytoma is a rare disease with incidence of 1-6 per million populations per year. ${ }^{4}$ Malignancy is detected in $10 \%$ of the PCC. ${ }^{5}$ The use of Pheochromocytoma of the Adrenal Gland Score (PASS) helps to diagnose malignant PCC from benign type. ${ }^{6}$ Biopsy of the metastatic lymph nodes, bone and liver and presence of chromaffin cells at non-chromaffin sites confirm the diagnosis. ${ }^{7}$ The diagnosis of PCC is by plasma or urinary catecholamine derivatives (metanephrines), urinary vinylmandelic-acid and iodine-labeled metaiodobenzylguanidine
(I-MIBG) level. In our patient the tumor was bigger in size, histopathological diagnosis of metastasis from lymph nodes and normal catecholamine level without any active secretion from the adrenal gland. Hence surgery followed by chemo and radiotherapy are the preferred management. ${ }^{8}$ The perioperative risk was very high. We adopted the strategy of managing hemodynamics and vital parameters for PCC, $\mathrm{CAD}$ and major long duration surgery.

The combined lesion of malignant pheochromocytoma with CAD is scanty in literature. ${ }^{1}$ The combined surgery of adrenal tumor excision and CABG is an option to minimize hospital stay. ${ }^{1}$ In our case the CABG was planned as second stage to avoid excess bleeding after heparin administration and the 2 nd point in favor of us that the hemodynamic were stable with no intra and postoperative MI. Intraoperative surgical inspection detected no intraluminal extension to IVC. The discrepancy between surgical finding and CT scan plus TEE reports regarding the IVC involvement may be due to the close vicinity of the tumor surrounding the walls of IVC which had created shadow imprint in the images.

This index case is an example of cardiac patient for non-cardiac surgery. ${ }^{2}$ As per revised cardiac risk index (RCRI) calculation, the perioperative risk of MI, cardiac arrest, or death 30 days after surgery of the patent was 8.1$12.6 \% .^{3}$ The surgery was planned as semi-urgent with extensive planning in cardiovascular thoracic setup for both facility for CPB and CABG. These back-ups or preparedness are needed to manage the patients at high risk in the moments of catastrophe during non-cardiac surgery. It save the life of patient at golden moment and reduces the perioperative morbidity and mortality.

To summarize malignant pheochromocytoma combined with $\mathrm{CAD}$ is an extremely rare case. The presence of extension of tumor to intra-vascular structures creates extra risk and necessitates special setup of cardiopulmonary bypass support to perform CABG and vascular surgery. Stabilization of blood pressure, balancing myocardial oxygen demand supply ratio, replacement of blood loss, temperature regulation and monitoring of cardiac parameters can prevent perioperative $\mathrm{MI}$ in the setting of a major non-cardiac surgery. Preparedness and team work is the key to success.

\section{Source of funding}

None.

\section{Conflict of interest}

None.

\section{References}

1. Baillargeon JP, Pek B, Teijeira J, Poisson J, Van Rossum N, Langlois MF. Combined surgery for coronary artery disease and pheochromocytoma. Can J Anaesth 2000;47(7):647-52.

2. Cohn SL. The cardiac consult for patients undergoing noncardiac surgery. Heart 2016;102:1322-32.

3. Rachel S B, Eric K O, David S B, John M C. Revised Cardiac Risk Index as a Predictor for Myocardial 
Infarction and Cardiac Arrest Following Posterior Lumbar Decompression. Spine 2019;44:E187-E193

4. Manger WM. An overview of pheochromocytoma: history, current concepts, vagaries, and diagnostic challenges. Ann NY Acad Sci 2006;1073:1-20.

5. Strong VE, Kennedy T, Al Ahmadie H, Tang L, Coleman J, Fong Y, Brennan M, Ghossein RA. Prognostic indicators of malignancy in adrenal pheochromocytomas: clinical, histopathologic, and cell cycle/apoptosis gene expression analysis. Surg 2008;143:759-68.
6. Kulkarni MM, Khandeparkar SG, Deshmukh SD. Risk Stratification in Paragangliomas with PASS (Pheochromocytoma of the Adrenal Gland Scaled Score) and Immunohistochemical Markers. J Clin Diagn Res 2016;10(9):EC01-EC04

7. Eisenhofer G, Bornstein SR, Brouwers FM. Malignant pheochromocytoma: current status and initiatives for future progress. Endocr Relat Cancer 2004;11:423-36.

8. Adjallé R, Plouin PF, Pacak K, Lehnert H. Treatment of malignant pheochromocytoma. Horm Metab Res 2009;41(9):687-96. 